\title{
Imposex en Crassilabrum crassilabrum (Neogastropoda: Muricidae) en la Costa Central del Perú
}

\author{
Imposex in CRassilabrum CRaSSilabrum (NeOgastropoda: Muricidae) in the \\ Peruvian Central Coast
}

\author{
Rolf Rivas ${ }^{1}$, Renzo Pradel ${ }^{1}$, Soledad Hurtado ${ }^{1}$, Dayanne Cornelio ${ }^{1}$, Jorge Mendoza ${ }^{1}$, \\ Angélica Guabloche ${ }^{1}$, José Iannacone ${ }^{2,3}$, Lorena Alvariño ${ }^{1}$, Luz Castañeda ${ }^{1}$
}

\section{Resumen}

El imposex es un fenómeno que consiste en el desarrollo de caracteres sexuales masculinos sobre el sistema reproductivo de las hembras de caracoles gasterópodos por la contaminación por compuestos organoestañosos (OTs), como el tributil estaño (TBT). En el presente trabajo se evaluó el imposex en Crassilabrum crassilabrum Sowerby, 1834 (Neogastropoda: Muricidae) proveniente de la costa central del Perú, para lo cual se analizaron individuos colectados de la zona intermareal de Pucusana y Ancón, Lima. Se tomaron medidas biométricas y se calcularon los índices de imposex. El porcentaje de imposex en Ancón fue del 98\% y en Pucusana de 100\%. El índice de la longitud relativa del pene (RPLI) fue de 52.3\% y 44.5\% para Pucusana y Ancón, respectivamente. Una relación significativa fue encontrada entre la longitud de la conchilla y la longitud del pene en machos en ambas playas, pero no así en las hembras. Crassilabrum crassilabrum es un molusco con aplicación para emplearse como un bioindicador de componentes OTs en la costa central del Perú y en el Pacífico sudamericano.

Palabras clave: Crassilabrum crassilabrum; imposex; organoestañosos; Pacífico Sur; Perú; caracol

\section{AbStract}

The imposex is a phenomenon that consists in the development of masculine sexual characteristics on the reproductive system of the gastropod female snails by organotin compounds (OTs), like tributyl tin (TBT). In the present study, the imposex was evaluated

\footnotetext{
${ }^{1}$ Laboratorio de Ecología y Biodiversidad Animal (LEBA), Facultad de Ciencias Naturales y Matemática, Universidad Nacional Federico Villarreal, Lima, Perú

${ }^{2}$ Laboratorio de Invertebrados, Facultad de Ciencias Biológicas, Universidad Ricardo Palma (URP), Lima, Perú

${ }^{3}$ E-mail: joseiannaconeoliver@gmail.com
}

Recibido: 10 de enero de 2017

Aceptado para publicación: 29 de abril de 2017 
in Crassilabrum crassilabrum Sowerby, 1834 (Neogastropoda: Muricidae) from the central coast of Peru, for which individuals were collected from the intertidal zone of Pucusana and Ancon, Lima. Biometric measurements were taken and the rates of imposex were calculated. The percentage of imposex in Ancon was of $98 \%$ and in Pucusana of $100 \%$. The index of relative length of the penis (RPLI) was $52.3 \%$ and $44.5 \%$ for Pucusana and Ancon, respectively. A significant relationship was found between the length of the conchilla and the length of the penis in males on both beaches, but not in females. Crassilabrum crassilabrum is a mollusk with application to be used as a bioindicator of OT components in the central coast of Peru and in the South American Pacific.

Key words: Crassilabrum crassilabrum; imposex; organotin; South Pacific; Peru; snail

\section{INTRODUCCIÓN}

El imposex es la superposición de caracteres sexuales masculinos sobre los femeninos cuando sustancias químicas modifican los mensajes transmitidos por las hormonas en moluscos gasterópodos prosobranquios (Mensink et al., 2002; Stickle y Zhang, 2003; Chacón et al., 2007; Afsar et al., 2015). Las sustancias que actúan de esta manera se les da el nombre de disruptores endocrinos $u$ hormonas medioambientales, y ocasionan impactos irreversibles sobre la salud del ecosistema (Chacón et al., 2007; El Mortaji et al., 2011; Guzmán y Ramírez, 2012). El imposex puede impedir la reproducción en las hembras de los caracoles llevando a la desaparición de la especie a escala local y regional (Bryan y Gibbs, 1986; Gibbs y Bryan, 1996; Guabloche et al., 2013).

Se conocen más de 260 especies de gasterópodos a nivel mundial que presentan imposex (Castro et al., 2012). Este fenómeno se ha asociado principalmente a compuestos químicos organoestañosos (OTs) a base de tributilestaño o TBT, utilizados como biocidas en las pinturas antiincrustantes de los cascos de las embarcaciones (Gagné et al., 2003; Rodríguez, 2010; Castro et al., 2012; Afsar et al., 2015; Artifon et al., 2016).

Se han detectado daños subletales y letal es en diversos componentes de la bi ota acuática por acción del TBT a partir de 0.4 ng de TBT L-1 en el ambiente acuático (Martinez-Llado et al., 2007; El Mortaji et $a l ., 2011)$. Estos daños incluyen toxicidad aguda, alteraciones en el ARN, neurotoxicidad, teratogenia e inmunotoxicidad. El TBT es uno de los xenobióticos más tóxicos y prioritarios producidos e introducidos deliberadamente en el ambiente acuático (IMO, 2008; Afsar et al., 2015; Cacciatore et al., 2016).

Se han incrementado y diversificado las actividades pesqueras en las últimas décadas en el Perú, lo cual conlleva a un mayor número de embarcaciones que utilizan pinturas antiincrustantes, las cuales aumentan las concentraciones de OTs en zonas con intensa actividad marítima, como puertos, marinas y astilleros (Castro et al., 2005; Lee et al., 2006; Aguilar, 2008; Cañete et al., 2015; Primost et al., 2015). Esta situación genera la necesidad de buscar nuevos biomarcadores para detectar y cuantificar los daños generados al ecosistema por el TBT (Artifon et al., 2016; Cacciatore et al., 2016). El imposex se puede disminuir si se toma en cuenta a la normatividad internacional que impide el uso de pinturas con TBT en embarcaciones mayores de $25 \mathrm{~m}$ de eslora. La normativa del IMO (Organización Marítima Internacional) del 2008 prohíbe el uso del TBT aplicado como pintura antifouling en los cascos de las naves (IMO, 2008; Cañete et al., 2015). 


\section{Crassilabrum crassilabrum} (Sowerby, 1834) (Muricidae) es una especie de caracol marino que habita la zona intermareal, y abarca una distribución latitudinal que va desde Pucusana, Perú, hasta los $46^{\circ} \mathrm{S}$ en el sur de Chile (Romero y Valdebenito, 2002).

En el Perú solo hay estudios de imposex en dos especies de caracoles marinos: Thaisella chocolata (Duclos, 1832) (Castro y Fillman, 2012; Chumbimune y Ponce, 2015) y Xanthochorus buxea (Broderip, 1833) (Guabloche et al., 2013). El caracol C. crassilabrum sería la tercera especie bioindicadora de imposex en la costa central del Perú, en dos playas de Lima: Playa Hermosa, distrito de Ancón y Playa de Pucusana, distrito de Pucusana. Es así que el objetivo de esta investigación fue evaluar el imposex en C. crassilabrum (Neogastropoda: Muricidae) en la costa central del Perú.

\section{Materiales y Métodos}

\section{Área de Estudio}

Playa Pucusana $\left(12^{\circ} 28^{\prime} 43^{\prime \prime}\right.$ LS, $76^{\circ} 47^{\prime} 44^{\prime \prime}$ LO) es una caleta de pescadores y un balneario que está ubicado a $60 \mathrm{~km}$ al sur de Lima. Es una de las playas que conforman el balneario de Pucusana, ubicada en el distrito del mismo nombre. El balneario tiene varias playas cuyas características son la tranquilidad y frialdad de sus aguas. Frente al litoral se encuentran diversas embarcaciones: yates, botes de pesca y turismo. Además, se realizan deportes acuáticos como el buceo.

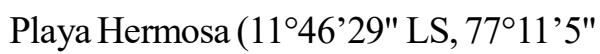
LO) es un puerto natural que está ubicado $43 \mathrm{~km}$ al norte de Lima. Es una de las playas que conforman el balneario de Ancón, ubicada en el distrito del mismo nombre. Frente al litoral se encuentran varias lanchas y barcos que desempeñan actividades pesqueras.

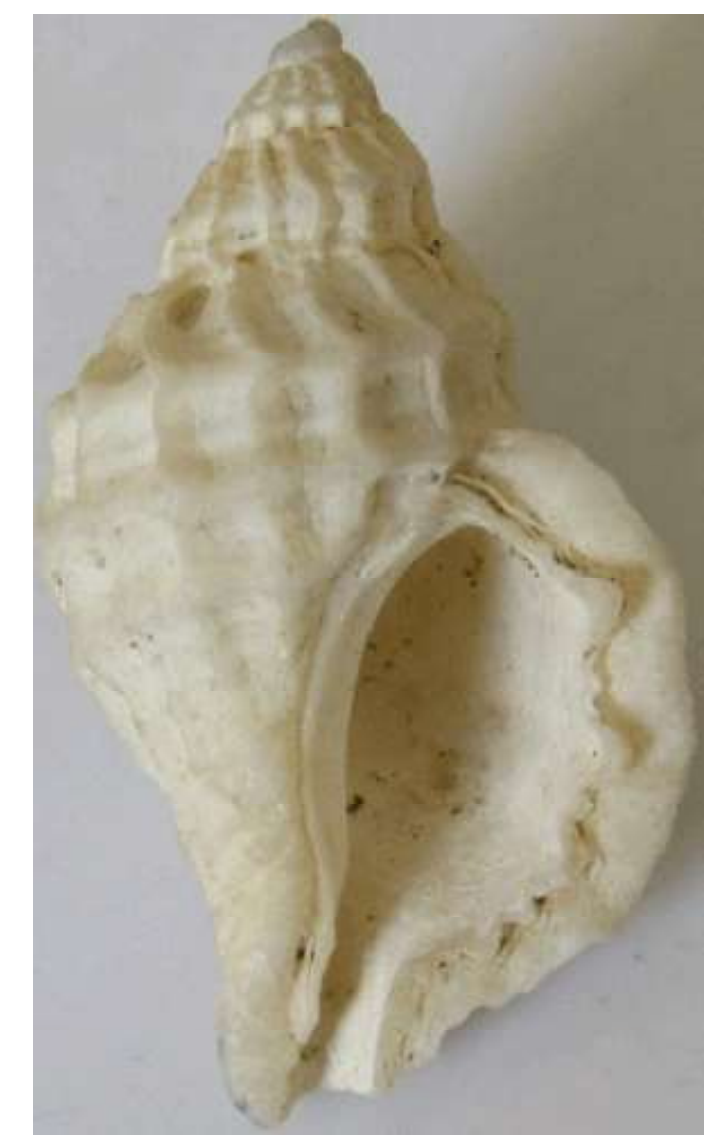

Figura 1. Espécimen de Crassilabrum crassilabrum (Muricidae)

\section{Crassilabrum crassilabrum}

Entre diciembre de 2014 y enero de 2015 se colectaron al azar 82 y 100 individuos de C. crassilabrum en el balneario de Pucusana y en Ancón, respectivamente. Se colocaron en baldes de plástico de $4 \mathrm{~L}$ con agua marina para su mantenimiento en vivo hasta su transporte al laboratorio. La toma de muestra se realizó a mano en la zona intermareal durante la bajamar. La especie fue identificada empleando las claves taxonómicas de Aldea y Valdovinos (2005) (Figura 1).

\section{Imposex}

Los organismos fueron narcotizados con una solución de $\mathrm{MgCl}_{2}$ al $3.5 \%$ durante $3 \mathrm{~h}$ (Castro y Fillman, 2012). La longitud de la 
conchilla (LC) fue medida desde el ápex hasta la base de la conchilla con un vernier $( \pm 0.01$ $\mathrm{mm}$ de precisión). Las conchillas fueron fraccionadas y removidas para el análisis de los tejidos blandos.

La identificación del sexo se basó en la presencia o ausencia de la glándula de la cápsula y ovarios para las hembras, y testículos y vesícula seminal para los machos (Gravel et al., 2006; Collado et al., 2010; Cuevas et al., 2011). El radio sexual fue determinado en base a la relación (Hembras $[\mathrm{H}] /$ Machos [M]) (El Mortaji et al., 2011; DomínguezOjeda et al., 2015). Se registró la longitud del pene (LP, en $\mathrm{mm}$ ) en machos y en hembras con imposex.

Los niveles de imposex fueron evaluados usando los siguientes índices: porcentaje de imposex en las hembras (I\%), índice de LP de las hembras (FPL, por sus siglas en inglés) y el índice de severidad relativo de LP (RPLI) o índice de Oehlman (Gibbs et al., 1987; Oehlman, 1996; Rodríguez, 2010; Cuevas et al., 2011; Castro y Fillman, 2012). Adicionalmente, para minimizar el efecto del tamaño en los parámetros de imposex, los índices de severidad FPL y RPLI fueron estandarizados con la longitud de la conchilla usando las ecuaciones indicadas por Castro y Fillmann (2012).

\section{Análisis Estadístico}

La normalidad de los datos (LC y LP) fue verificada usando la prueba de Kolmogorov-Smirnov. Para analizar si la longitud del pene es afectada por la longitud de la conchilla fue empleada una regresión y una correlación lineal entre estos parámetros. También se realizó la prueba de t- Student para verificar si existen diferencias significativas entre la LC y la LP según sexo. Todos los análisis estadísticos fueron llevados a cabo con el paquete estadístico PAST y Minitab 17 , con un nivel de significancia de 0.05 .

\section{Resultados}

Los cuadros 1 y 2 presentan los datos biométricos y los parámetros de imposex ( $\mathrm{I} \%$, RPLI, FPL, FPL estándar y RPLI estándar) de $C$. crassilabrum en la costa central del Perú. Se encontró un mayor porcentaje de caracoles machos que hembras (Pucusana: ratio sexual $=1.21 ;$ Ancón: ratio sexual $=$ 1.38). La longitud de las conchillas (LC) fluctuó entre 13.5 y $29.1 \mathrm{~mm}$ para Pucusana y entre 17.5 y $31.7 \mathrm{~mm}$ para Ancón, encontrándose diferencias significativas entre sexos para Pucusana $(\mathrm{t}=2.69 ; \mathrm{p}=0.008)$ y Ancón $(\mathrm{t}=0.72 ; \mathrm{p}=0.049)$ (Cuadro 1). Los niveles de imposex para Pucusana y Ancón fueron de 100 y $98.3 \%$, respectivamente, evidenciado por el desarrollo de un pseudopene detrás del tentáculo derecho.

La LP de los machos presentó un mayor rango de variación en ambas playas, mientras que en las hembras con imposex el rango fue más estrecho. Sin embargo, se encontraron diferencias significativas entre machos y hembras para ambas playas (Pucusana: $\mathrm{t}=10.97 ; \mathrm{p}<0.001$; Ancón: $\mathrm{t}=14.14 ; \mathrm{p}<0.001$ ) (Cuadro 2).

Una correlación positiva y estadísticamente significativa fue observada entre la LC y LP en los machos en Pucusana $(r=0.58$, $\mathrm{p}=0.002)$ y en Ancón ( $\mathrm{r}=0.71, \mathrm{p}=0.0001)$ (Figuras 2 y 3). En el caso de las hembras se notó un bajo coeficiente de correlación con ausencia de significancia estadística en Pucusana $(\mathrm{r}=0.37, \mathrm{p}=0.59)$ y en Ancón $(\mathrm{r}=0.11, \mathrm{p}=0.40)$.

\section{Discusión}

Los datos revelan que el fenómeno de imposex afecta poblaciones de $C$. crassilabrum en ambas localidades de Lima, Perú. Esto puede sugerir la presencia del 
Cuadro 1. Longitud de la conchilla $(\mathrm{mm})$ de Crassilabrum crassilabrum (Muricidae) colectados en dos playas (Pucusana y Playa Hermosa) de Lima, Perú

\begin{tabular}{lcccccc}
\hline \multirow{2}{*}{ Localidad } & \multicolumn{3}{c}{ Machos } & \multicolumn{3}{c}{ Hembras } \\
\cline { 2 - 7 } & $\mathrm{n}$ & Prom. \pm d.e. & Rango & $\mathrm{n}$ & Prom. \pm d.e. & Rango \\
\hline Pucusana & 37 & $21.2 \pm 2.5$ & $13.5-26.4$ & 45 & $21.7 \pm 2.9$ & $15.0-29.1$ \\
Ancón & 42 & $22.7 \pm 2.2$ & $17.5-26.8$ & 58 & $24.1 \pm 2.7$ & $19.2-31.7$ \\
\hline
\end{tabular}

Cuadro 2. Datos biométricos y de imposex ${ }^{1}$ en Crassilabrum crassilabrum (Muricidae) colectados en dos playas: Pucusana ( 37 machos y 45 hembras) y Playa Hermosa (42 machos y 58 hembras), Lima, Perú

\begin{tabular}{|c|c|c|c|c|c|c|c|c|}
\hline \multirow[t]{2}{*}{ Localidad } & \multicolumn{2}{|c|}{ Machos } & \multicolumn{2}{|c|}{ Hembras } & \multirow{2}{*}{ RPLI } & \multirow{2}{*}{$\begin{array}{l}\text { FPL } \\
\text { estand }\end{array}$} & \multirow{2}{*}{$\begin{array}{l}\text { RPLI } \\
\text { estand }\end{array}$} & \\
\hline & MPL \pm d.e & Rango & FPL \pm d.e & Rango & & & & \\
\hline Pucusana & $5.4 \pm 0.8$ & $3.8-7.1$ & $3.3 \pm 0.6$ & $1.6-4.3$ & 52.3 & 0.1 & 52.2 & 100 \\
\hline Ancón & $5.1 \pm 1.5$ & $2.1-8.4$ & $2.3 \pm 1.0$ & $0.8-6.4$ & 44.5 & 0.1 & 41.9 & 98.3 \\
\hline
\end{tabular}

${ }^{1}$ MPL: Promedio de la longitud del pene de los machos; FPL: Promedio de la longitud del pene de las hembras; RPLI: Índice relativo a la longitud del pene; \%: Porcentaje de imposex; estand = estandarizado

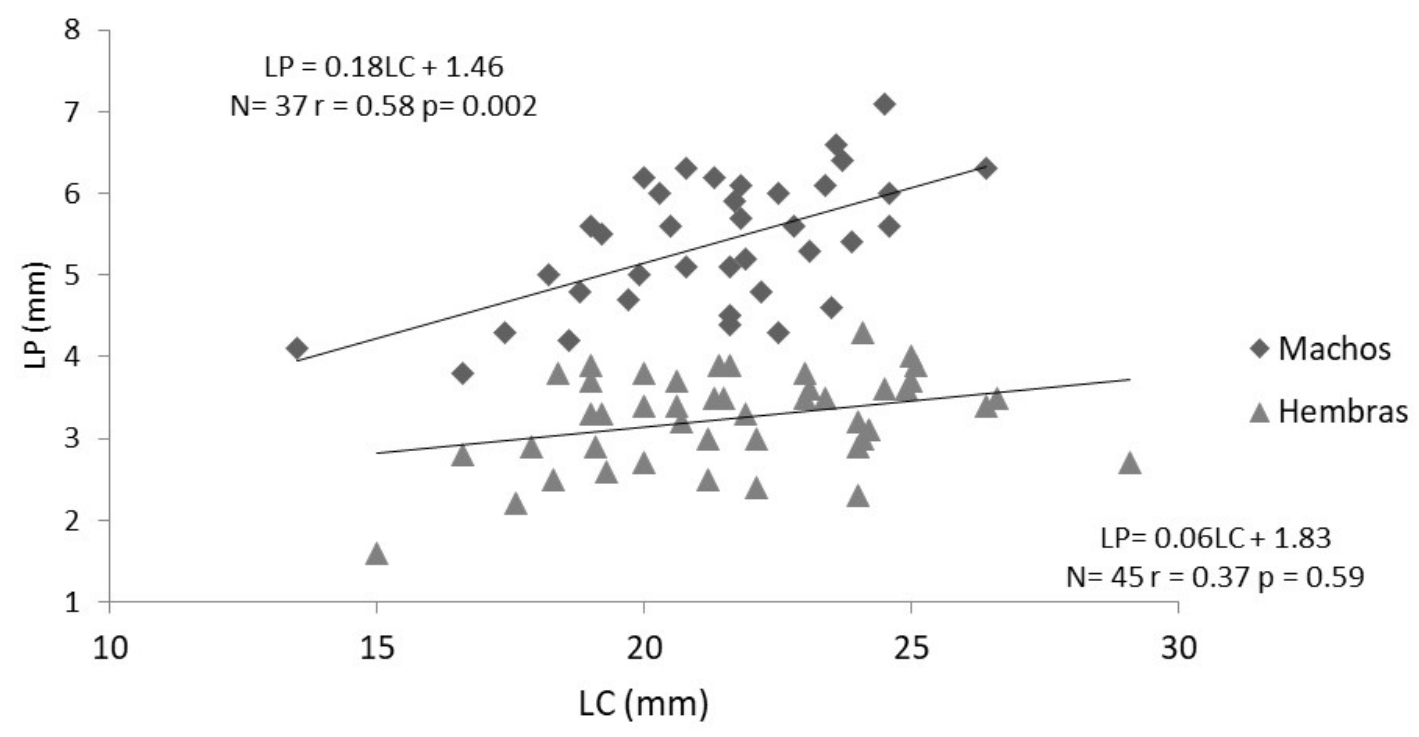

Figura 2. Relación entre longitud de la conchilla (LC) y la longitud del pene (LP) en machos y hembras de Crassilabrum crassilabrum (Muricidae), Playa Pucusana, Pucusana (Lima, Perú) 


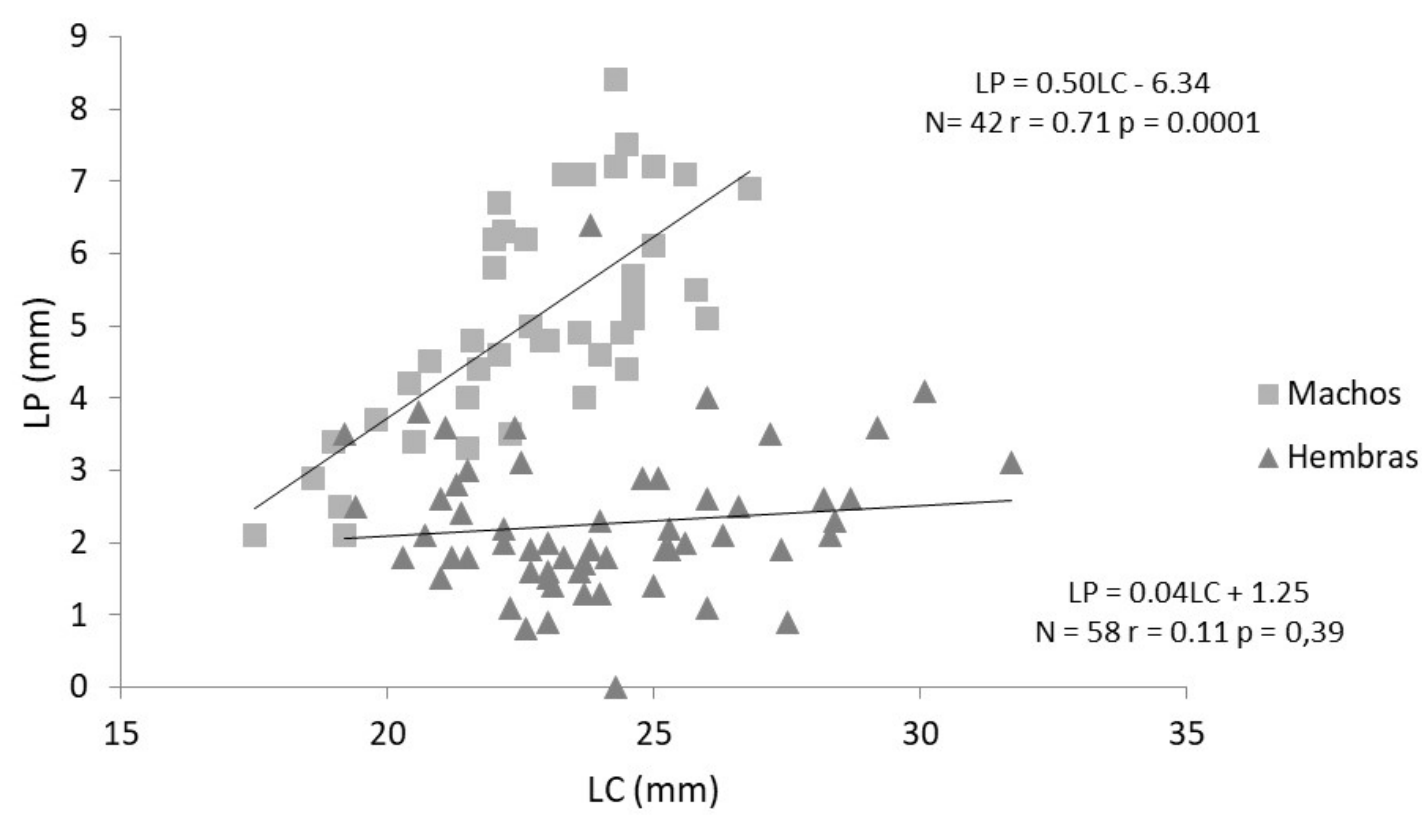

Figura 3. Relación entre longitud de la conchilla (LC) y la longitud del pene (LP) en machos y hembras de Crassilabrum crassilabrum (Muricidae), Playa Hermosa, Ancón (Lima, Perú)

contaminante (TBT) en el ambiente marino (Lima-Verde et al., 2010; Domínguez-Ojeda et al., 2015). Este mismo patrón ha sido observado en otros caracoles marinos, principalmente murícidos (El Mortaji et al., 2011; Cañete et al., 2015; Primost et al., 2015; Batista et al., 2016). Se han registrado altas correlaciones dosis-dependientes entre las fuentes de TBT y la presencia e imposex, lo cual se ha confirmado en varios estudios por los análisis químicos de agua, sedimento, y tejidos del caracol (El Mortaji et al., 2011; Batista et al., 2016); de allí que se pude considerar que altos niveles de TBT estarían ocurriendo en la costa central marina del Perú.

Se registra imposex en C. crassilabrum para ambas zonas con altas prevalencias de 98\% en Playa Hermosa y 100\% en la Playa de Pucusana. En esta última, el tráfico marítimo, por la presencia de embarcaciones pesqueras, es mayor y con mayor densidad (Primost et al., 2015; Batista et al., 2016), además de no mantener un control sobre las pinturas con OTs. Ambas playas tienen una geografía que disminuye la cinética y el hidrodinamismo de la marea, favoreciendo la sedimentación de OTs. Los resultados obtenidos en estas playas de Lima evidencian la necesidad de ejercer mayor control aplicado a la normatividad vigente sobre el uso de este tipo de compuestos (TBT) para prevenir una alteración irremediable del ecosistema por problemas de reproducción en poblaciones afectadas por imposex (Chacon et al., 2007; El Mortaji et al., 2011; Primost et al., 2015; Batista et al., 2016).

La baja correlación entre LP y LC en el caso de las hembras se podría deber a que la LP depende más de la exposición al contaminante TBT que de la longitud de la conchilla. Patrones similares han sido previamente registrados para otros caracoles murícidos (Vasconselos et al., 2011; Castro y Fillman 2012; Guabloche et al., 2013; DomínguezOjeda et al., 2015). 
El índice de imposex FPLI es mayor en la Playa de Pucusana. Este índice está estrechamente relacionado con las concentraciones de OTs biodisponibles en el medio, lo cual reafirma la suposición de que la Playa de Pucusana presenta mayor contaminación por OTs (Batista et al., 2016). Se ha postulado que valores de RPLI superiores a $25 \%$ indicarían que la población de caracoles tiene altos riesgos de disminuir (Cañete et al., 2015). En el presente trabajo se han observado valores de severidad de imposex (RPLI) entre 44.5 y $52.3 \%$, por los que el caracol marino C. crassilabrum tendría altos riesgos de disminuir sus densidades poblacionales (Cañete et al., 2015).

Al ser los índices de imposex tan altos en $C$. crassilabrum, se sugiere usar esta especie como bioindicadora o centinela para realizar monitoreos y detectar «hot spots» (puntos clave) de contaminantes de la familia de los OTs en la costa central peruana, y en especial en programas de manejo costero marino (El Mortaji et al., 2011; DomínguezOjeda et al., 2015; Cacciatore et al., 2016). Esto es un asunto de preocupación a nivel mundial ya que se sigue empleando TBT en varios países a pesar de la prohibición mundial (Polanía, 2010; Batista et al., 2016). El empleo del imposex para evaluar la calidad ambiental marina por contaminación por TBT es un protocolo rápido, barato y sus resultados son fáciles de interpretar (Polanía, 2010).

A nivel mundial, las embarcaciones de gran envergadura han sido objeto de interés al ser la principal fuente de contaminación por TBT (Afsar et al., 2015). Sin embargo, Batista et al. (2016) señalan que para Sudamérica las embarcaciones medianas y pequeñas (botes de pesca y recreativos) serían la principal fuente de TBT. Esta última afirmación podría explicar la alta presencia y severidad de imposex en C. crassilabrum en Ancón y Pucusana, indicador indirecto de la presencia de TBT.

Sería importante comprobar si el fenómeno de imposex en caracoles marinos es reversible a nivel poblacional al trasladar a
C. crassilabrum a ambientes no perturbados o pristinos, y si emplear pinturas con otros compuestos biocidas ocasiona un menor riesgo al ecosistema marino.

\section{Literatura Citada}

1. Afsar N, Siddiqui G, Ayub Z. 2015. Bioindicator Thais carinifera (mollusca, Gastropoda): imposex, response and consequences along the Pakistan coast during the period from 1993 to 2012 . Braz J Oceanogr 63: 115-124. doi: 10.1590/S1679-85592015082006302

2. Aguilar FPG 2008. Evaluación ambiental en zonas marino costeras del Perú. 2002, 2003 y 2004. Inf Inst Mar Peru 35: 7-74.

3. Aldea C, Valdovinos C. 2005. Moluscos del intermareal rocoso del Centro-Sur de Chile $\left(36^{\circ}-38^{\circ} \mathrm{S}\right)$ : taxonomía y clave de identificación. Gayana 69: 364-396. doi: 10.4067/S0717-65382005000200014

4. Artifon V, Castro IB, Fillman G. 2016. Spatiotemporal appraisal of TBT contamination and imposex along a tropical bay (Todos os Santos Bay, Brazil). Environ Sci Pollut Res 23: 16047-16055. doi: 10.1007/s11356-016-6745-7

5. Batista RM, Castro IB, Fillman G. 2016. Imposex and butylin contamination still evident in Chile after TBT global ban. Sci Tot Env 566-567: 446-453. doi: 10.1016/j.scitoenv.2016.05.039

6. Bryan PE, Gibbs GW. 1986. Reproductive failure in populations of the dogwhelk, Nucella lapillus, caused by imposex induced by tributyltin from antifouling paints. J Mar Biol Assoc UK 66: 767-777. doi: 10.1017/S0025315400048414

7. Cacciatore F, Noventa S, Antonini C, Formalewicz M, Gion C, Berto D, Gabellini M, Brusá RB. 2016. Imposex in Nassarius nitidus (Jeffreys, 1867) as a possible investigative tool to monitor butyltin contamination according to the Water Framework Directive: a case study in the Venice Lagoon (Italy). 
Ecotox Env Saf 138. doi: 10.1016/ j.ecoenv.2015.12.039

8. Cañete JI, Osorio C, Huaquín L. 2015. Nuevos registros de imposex en muricidos de la zona austral de Sudamérica (Estrecho de Magallanes). Cienc Tecnol Mar 36: 81-90.

9. Castro I, Perina F, Fillmann G. 2012. Organotin contamination in South American coastal areas. Environ Monit Assess 184: 1781-1799. doi: 10.1007/ s10661-011-2078-7

10. Castro IB, Braga ARC, RochaBarreira CA. 2005. Altos índices de imposex em Stramonita rustica (Mollusca: Gastropoda) em áreas portuárias dos estados de Alagoas e Sergipe, Brasil. Trop Oceanogr Recife 33: 121-128,

11. Castro IB, Fillman G. 2012. High tributyltin and imposex levels in the commercial muricid Thais chocolata from two Peruvian harbor areas. Environ Toxicol Chem 31: 955-960. doi: 10.1002/ etc. 1794

12. Chacón O, Cuevas F, La Fuente C, Díaz F, Huaquín L. 2007. Disrupción endocrina e imposex. Avances Cienc Vet 22: 42-48.

13. Chumbimune OLM, Ponce MZJ. 2015. Monitoreo de contaminación por tributilestaño (TBT) en Puertos de Paraca, Ica (Perú), mediante el fenómeno de imposex en Stramonita chocolata. Científica 12: 222-230.

14. Collado G, Osorio C, Retamal M. 2010. Imposex en los caracoles marinos Acanthina monodon (Pallas, 1774) y Nassarius coppingeri E.A. Smith, 1881 en el sur de Chile. Cienc Tecnol Mar 33: 67-76.

15. Cuevas N, Larreta J, Rodríguez JG, Zorita I. 2011. A visual guideline for the determination of imposex in Nassarius reticulatus and Nassarius nitidus. Rev Inv Mar AZTI-Tecnalia 18: 134-152.

16. Domínguez-Ojeda D, Patrón-Soberano OA, Nieto-Navarro JT, RobledoMarenco ML, Velásquez-Fernández JB. 2015. Imposex in Plicopurpura pansa (Neogastropoda: Thaididae) in Nayarit and Sinaloa, Mexico. Rev Mex Biodiv 86: 531-534. doi: 10.1016/ j.rmb.2015.04.018

17. El Mortaji H, Elkhiati N, Benhra A, El Haimeur B, Bouhallaoui $M$, Benbrahim S, Kabine M, Ramdani M. 2011. Imposex in Stramonita haemastoma (Gastropoda: Muricidae) along the Atlantic and Mediterranean coast of Morocco. Bull Inst Sci Rabat 33: 13-18.

18. Gagné F, Blaise CC, Pellerin J, Pelletier E, Douville M, GauthierClerc S, Viglino L. 2003. Sex alteration in soft-shell clams (Mya arenaria) in an intertidal zone of the Saint Lawrence river (Quebec, Canada). Comp Biochem Physiol Part C: 134: 189-198.

19. Gibbs PE, Bryan GM, Pascoe PL, Burt GR. 1987. The use of dog-whelk Nucella lapillus, as an indicator of tributyltin (TBT) contamination. J Mar Biol Assoc UK 67: 507-523. doi: 10.1017/ S0025315400027260

20. Gibbs PE, Bryan GW. 1996. TB Tinduced imposex in neogastropod snails: masculinization to mass extinction. In: de Mora SJ (ed). Tributyltin: case study of an environmental contaminant, Cambridge, UK: Cambridge University Press. $\mathrm{p}$ 212-236.

21. Gravel P, Johanning K, McLachlan J, Vargas JA, Oberdörster E. 2006. Imposex in the intertidal snail Thais brevidentata (Gastropoda, Muricidae) from the Pacific coast of Costa Rica. Rev Biol Trop 54(Supl 1): 21-26. doi: 10.15517/rbt.v54i1.26829

22. Guabloche A, Alvarez J, Rivas R, Hurtado S, Pradel R, Iannacone J. 2013. Imposex en el caracol marino Xanthochorus buxea (Broderip, 1833) (Muricidae) del Pacífico Sudamericano. Biologist (Lima) 11: 237-249.

23. Guzmán MC, Ramírez P. 2012. Disfunción endocrina debido a contaminantes ambientales. Elementos 87: 19-25.

24. [IMO] International Maritime Organization. 2008. Anti-fouling systems. [Internet]. Disponible en: http:// 
www.imo.org/en/OurWork/Environment/Anti-foulingSystems/Pages/ Default.aspx

25. Lee CC, Hsieh CY, Tien CJ. 2006. Factors influencing organotin distribution in different marine environmental compartments and their potential health risk. Chemosphere 65: 547-559. doi: 10.1016/j.chemosphere.2006.02.037

26. Lima-Verde FB, Castro IB, RochaBarreira CA. 2010. Imposex occurrence in Nassarius vibex from South America: a potential bioindicator in estuarine environments. Mar Biodivers Rec 3: e30. doi: 10.1017/ S1755267209990716

27. Martinez-Llado X, Gibert O, Marti V, Diez S, Romo J, Bayona J. de Pablo JM. 2007. Distribution of polycyclic aromatic hydrocarbons (PAHs) and tributyltin (TBT) in Barcelona harbour sediments and their impact on benthic communities. Environ Pollut 149: 104113. doi: 10.1016/j.envpol.2006.11.020

28. Mensink B, Kralt H, Vethaak AD, Ten Hallers-Tjabbes C, Koeman JH, Van Hattun B, Boon JP. 2002. Imposex induction in laboratory reared juvenile Buccinun undatun by tributyltin (TBT). Environ Toxicol Pharmacol 11: 49-65.

29. Oehlmann J, Fioroni P, Stroben E, Markert B. 1996. Tributyltin (TBT) effects on Ocinebrina aciculata (Gastropoda: Muricidae): imposex development, sterilization, sex change and population decline. Sci Tot Env 188: 205-223. doi: 10.1016/0048-9697(96)05173-X

30. Polanía J. 2010. Indicadores biológicos para el monitoreo de puertos en Colombia. Rev Gestión Ambiente 13: 75-86.

31. Primost MA, Averbuj A, Bigatti G. 2015. Variability of imposex development and reproductive alterations in the Patagonian gastropod Buccinanops globulosus inhabiting a polluted harborarea. Rev Mus Argentino Cienc Nat 17: 167-171.

32. Rodríguez F. 2010. Imposex en la Laguna de Términos, Campeche, México. UDO Agric 10: 141-149.

33. Romero MS, Valdebenito EL. 2002. Larvas veliger de gastropodos Prosobranchia provenientes de Punta de Lobos, Cuarta Región, Chile. Rev Chil Hist Nat 75: 491-514. doi: 10.4067/S0716078X2002000300003

34. Stickle W, Zhang Z. 2003. Long-term trends in imposex in six populations of Stramonita haemastoma. Bull Mar Sci 72: 685-694.

35. Vasconcelos P, Moura P, Castro M, Gaspar M. 2011. Size matters: importance of penis length variation on reproduction studies and imposex monitoring in Bolinus brandaris (Gastropoda: Muricidae). Hydrobiologia 661: 363-375. doi: $10.1007 / \mathrm{s} 10750-010-0544-9$ 